\title{
EFEITO DO MÉTODO PILATES EM MULHERES GESTANTES - ESTUDO CLÍNICO CONTROLADO E RANDOMIZADO
}

\section{EFFECT OF THE PILATES METHOD IN PREGNANT WOMENS - STUDY CLINICAL RANDOMIZEID CONTROLLED}

\author{
Bianca Spitzner Justino ${ }^{1}$; Wagner Menna Pereira ${ }^{2^{*}}$ \\ ${ }^{1}$ Faculdade Guairacá, Departamento de Fisioterapia, Guarapuava, Paraná, Brasill, ${ }^{2 *}$ Faculdade \\ Guairacá/SESG, Departamento de Fisioterapia, Guarapuava, Paraná, Brasil \\ *Autor correspondente: Rua Senira Baroni Kuster, 379, Alto da XV, CEP: 85.065-045; \\ Guarapuava, PR. E-mail: wagner.fisio@hotmail.com
}

\begin{abstract}
RESUMO
Durante o período gestacional, a mulher passa uma sequência de adaptações fisiológicas, morfológicas, sociais e emocionais. Dentro dessas alterações, ocorrem desequilíbrios no sistema articular devido ao aumento da massa corpórea e de suas dimensões, gerando perturbação do centro de gravidade (CG) e maior oscilação do centro de força (CF). Avaliar os efeitos do Método Pilates na oscilação do centro de massa corporal e na dor lombar de gestantes. Trata-se de um estudo clínico controlado e randomizado em que participaram 15 mulheres entre a $13^{\mathrm{a}}$ e $36^{\mathrm{a}}$ semana gestacional com idade de 18 a 40 anos, divididas em 2 grupos, sendo um grupo controle, composto por 8 mulheres, e um grupo pilates, composto por 7 mulheres que realizaram 10 intervenções em solo, 2 vezes por semana, durante 5 semanas. O processo de avaliação antes e depois dos exercícios foi realizado através da Plataforma Estabilométrica e preenchimento da Escala Visual Analógica da Dor (EVA). Diante da análise estatística, o presente estudo demonstrou efeitos positivos significantes na comparação da redução da dor lombar pela EVA do grupo Pilates $(p=0,001)$ em relação ao grupo Controle $(\mathrm{p}=0,1394)$. Quanto a variável na Oscilação Estabilométrica, não houve diferença estatisticamente significante, sendo $\mathrm{p}=0,680$. O programa de exercícios do Método Pilates evidenciou redução na intensidade de dor lombar das gestantes, entretanto, não foi constatada melhora estatisticamente significante na oscilação do centro de massa corporal. Palavras-Chaves: Gestantes; Equilíbrio Postural; Dor Lombar; Técnica de Exercício e de Movimento.
\end{abstract}

\begin{abstract}
During pregnancy, the woman passes a sequence of physiological, morphological, social and emotional adjustments. Within these changes occur imbalances in the joint system due to increased body mass and its dimensions, creating disturbance of the center of gravity (CG), and greater sway the force center (CF). To assess the effects of Pilates in the oscillation of the body mass center and back pain of pregnancy. This is a controlled clinical randomized study who participated 15 women between 13 and 36 gestational week aged 18 to 40 , divided into 2 groups, one control group, consisting of 8 women and pilates group, composed of seven women who underwent 10 interventions on the ground, 2 times a week for 5 weeks. The evaluation process before and after exercise was performed by stabilometric platform and fill the Visual Analog Pain Scale (VAS). Before the statistical analysis, the present study demonstrated significant positive effects in comparison to the reduction of low back pain by Pilates group VAS $(p=0.001)$ compared to control group $(p=0.1394)$. As the variable in stabilometric Oscillation, there was no statistically significant difference, $p=0.680$. The exercise program Pilates showed reduction in the intensity of low back pain in pregnant women, however, it found no statistically significant improvement in the oscillation of the body center of mass.
\end{abstract}

Keywords: Pregnant Women; Balance Postural; Low Back Pain; Exercise Movement Techniques. 


\section{INTRODUÇÃO}

A gravidez é um processo fisiológico compreendido pela sequência de adaptações dos mais variados sistemas a partir da fertilização, advindas de mudanças hormonais e biomecânicas que provocam alterações estruturais na estática e dinâmica no corpo da gestante, as quais são fundamentais para regular o metabolismo materno, ajudar no crescimento fetal e preparar a mulher para o momento de trabalho de parto e lactação (MANN, et. al., 2011; BARBOSA; SILVA; MOURA, 2011). Dentre essas alterações, cita-se a intensa ação dos hormônios progesterona, estrógeno, cortisol e relaxina. O hormônio relaxina, produzido pelo corpo lúteo, é considerado o principal responsável pela frouxidão ligamentar durante a gravidez, o que permite que a sínfise púbica e a articulação sacro-ilíaca tornem-se mais flexíveis para a passagem do feto, levando à redução da estabilidade pélvica (FIRMENTO, et. al., 2012; ALBINO, et. al., 2011).

Assim como a relaxina, outras alterações podem ser citadas durante o período gestacional. Dentre essas alterações, o aumento da carga e o desequilíbrio no sistema articular devido ao acréscimo da massa corpórea e de suas dimensões podem provocar perturbação do centro de gravidade (CG) e maior oscilação do centro de força (CF), que levam a um equilíbrio instável e influenciam na biomecânica da postura. Essa alteração pode aumentar o risco de quedas em gestantes, além de gerar desconfortos na coluna lombar, justificada pelas mudanças no equilíbrio postural que reflete em anteriorização do centro de gravidade e sobrecarga da musculatura da coluna lombar (RIBAS SI e GUIRRO ECO, 2007; CORTEZ, et al., 2012).

Para Gomes et. al., (2013) e Novaes, Shimo e Lopes (2006), a lombalgia pode ser baseada em três condições: dor lombar, dor pélvica posterior ou combinação de ambas. A dor lombar é um sintoma presente previamente à gestação, que se intensifica durante esse período, sendo observada diminuição da mobilidade da região lombar no exame clínico e dor à palpação da musculatura paravertebral lombar. A dor pélvica posterior é uma lombalgia característica da gestação, de natureza intermitente podendo gerar irradiação para os glúteos e membros inferiores, que causa dor e bloqueio de movimento durante a marcha e teste de provocação da dor pélvica posterior positivo.
Mediante as repercussões decorrentes do período gestacional, evidencia-se a necessidade de buscar métodos e instrumentos para identificação e avaliação de possíveis alterações posturais sucedidas da gestação (LIMA, et. al., 2011). Dentre esses métodos, destaca-se a avaliação da oscilação postural, que fornece informações a respeito das variáveis que influenciam o controle postural, contribuindo clinicamente para a identificação dos fatores relacionados às quedas $\mathrm{e}$ suas lesões associadas. Testes clínicos podem avaliar como as deficiências no centro de oscilação de massa corporal, nas forças e resistências musculares afetam as atividades diárias. Dentre os instrumentos utilizados para fins avaliativos cita-se a estabilometria, que se trata de um recurso para quantificação da oscilação corporal, por monitorar o deslocamento do Centro de Pressão (CP). O exame registra a oscilação contínua do corpo humano com o auxílio de uma plataforma de força, cujos deslocamentos ântero-posterior e médio-lateral são analisados quanto ao $\mathrm{CP}$, tanto na posição ereta estática ou dinâmica. A partir desse registro podem ser calculadas diversas variáveis relacionadas à oscilação postural (FERREIRA et. al., 2010).

Para a regulação do equilíbrio, o sistema necessita de informações sobre as posições relativas dos segmentos do corpo e da magnitude das forças atuando sobre o corpo (DUARTE, 2000; RODRIGUES et. $a l ., 2009)$. Trata-se de uma complexa integração das informações sensoriais ascendentes e descendentes, advindas dos sistemas visual, somatossensorial e vestibular, relativas à posição do corpo e à habilidade para gerar respostas motoras apropriadas para controlar o movimento corporal. Uma das maneiras de se recrutar esses sistemas de integração sensoriais é através de exercícios terapêuticos. Dentre os exercícios mais utilizados na prática clínica, cita-se o método Pilates, idealizado pelo alemão Joseph Hubertus Pilates (18801967), que proporciona um amplo benefício para o corpo humano, estimulando a circulação, melhorando a flexibilidade, a amplitude de movimento, ou seja, a postura e o condicionamento do corpo, auxiliando no controle físico e mental em geral. Os exercícios são adaptados conforme as condições físicas de forma individual (MANN, et. al., 2011; MARÉS, et. al., 2012; POSADZKI; LIZIS; DERENGOWSKA, 2011). Segundo Rodrigues et. al., (2010), o Método Pilates consiste em exercícios físicos cuja característica principal é o trabalho resistido e o alongamento dinâmico, 
realizados em conjunto com a respiração e respeitando os seguintes princípios: controle, precisão, centralização, fluidez de movimento, concentração e respiração.

Para gestantes, o fortalecimento deve priorizar a musculatura do assoalho pélvico, paravertebral lombar, a cintura escapular e, preferencialmente, envolver grandes grupos musculares, denominados de "powerhouse" (NASCIMENTO et. al., 2014).

Diante da frequente queixa de dor lombar e na tentativa de investigar os fatores posturais associados com as alterações do período gestacional, o presente trabalho tem por objetivo apresentar, por meio de uma abordagem fisioterapêutica, considerações a respeito da prática do método Pilates durante a gestação, com enfoque especial sobre o efeito no controle do centro de oscilação de massa do corpo e sua influência na dor lombar.

\section{MATERIAL E MÉTODO}

Realizou-se um estudo clínico controlado e randomizado, aprovado pelo Comitê de Ética da Universidade Estadual do Centro-Oeste, GuarapuavaPR, sob o protocolo ${ }^{\circ} 896.238 / 2014$, conforme a resolução 466/12 do Conselho Nacional em Saúde. A amostra constituiu-se por conveniência, composta por 15 gestantes entre a $13^{\mathrm{a}}$ e $36^{\mathrm{a}}$ semana gestacional que realizavam consulta de pré-natal no Posto de Saúde dos Bairros Santana e Santa Cruz, localizados no município de Guarapuava-PR, e divididas em 2 grupos. O Grupo Pilates, composto por 7 gestantes que realizaram exercícios de Pilates, e o Grupo Controle, composto por 8 gestantes. Para a distribuição e alocação dos grupos, foi realizada a randomização aleatória sem reposição pelo Software BioEstat. A coleta de dados foi realizada nas Clínicas Integradas Guairacá, entre os meses de abril a maio de 2015 , por meio de entrevista e exame físico individualizado. Foram incluídas voluntárias gestantes, maiores de 18 anos de idade, com queixas de desconforto lombar durante a gestação. Excluíram-se gestantes que apresentaram contraindicações clínicas ou obstétricas, problemas vestibulares; voluntárias que ainda não haviam completado a $12^{\mathrm{a}}$ semana gestacional e/ou estavam entre a $37^{\mathrm{a}}$ e $42^{\mathrm{a}}$ semana gestacional e que nunca tinham praticado nenhuma atividade física. Todas as voluntárias foram informadas sobre os procedimentos da pesquisa e assinaram o Termo de Consentimento Livre e Esclarecido - TCLE.

Ambos os grupos foram submetidos ao preenchimento de uma ficha cadastral, da Escala Visual Analógica da Dor (EVA), que se descreve como uma escala de 0 a 10 pontos graduada em centímetros, onde as voluntárias assinalaram conforme sua dor no momento, e realizaram uma avaliação com dados antropométricos. Todas as voluntárias passaram por uma avaliação estabilométrica por meio da plataforma da marca Arkipélago para obtenção dos valores da oscilação corporal pré-procedimento, sendo cada avaliação calibrada pelo próprio software Footwork do aparelho, através dos dados de peso, altura e tamanho dos pés das voluntárias. Em seguida as oscilações corporais foram avaliadas em área total de deslocamento do centro de gravidade $\left(\mathrm{cm}^{2}\right)$. Durante a coleta, as voluntárias foram orientadas a partir de uma posição inicial neutra, permanecerem estáticas, com os olhos fixados e boca semiaberta durante o período de 30 segundos. Foram realizadas avaliações de forma bipodal. Na tabela 1 estão expressos os valores antropométricos da amostra estudada.

Quadro 1. Dados antropométricos dos grupos expressos em média e desvio padrão

\section{QUADRO CARACTERÍSTICAS DOS GRUPOS}

\begin{tabular}{|c|c|c|c|c|} 
& IDADE & MASSA $(\mathrm{kgf})$ & ALTURA $(\mathrm{cm})$ & $\mathrm{IMC}\left(\mathrm{kg} / \mathrm{m}^{2}\right)$ \\
\hline $\begin{array}{c}\text { Grupo } \\
\text { Pilates }\end{array}$ & $23,5 \pm 4,84$ & $67,0 \pm 15,3$ & $1,61 \pm 0,06$ & $25,5 \pm 4,99$ \\
\hline $\begin{array}{c}\text { Grupo } \\
\text { Controle }\end{array}$ & $24,2 \pm 2,72$ & $71,1 \pm 7,72$ & $1,65 \pm 0,05$ & $25,8 \pm 1,83$ \\
\hline P Valor & 0,70 & 0,53 & 0,17 & 0,80 \\
\hline
\end{tabular}

Após a avaliação, foram realizados 10 atendimentos de Pilates duas vezes por semana, durante 60 minutos cada, em dias alternados por um período de cinco semanas. Os atendimentos constituíram-se de exercícios para fortalecimento, alongamento e relaxamento, através da utilização de bolas suíças, fitas elásticas, overballs e rolos. Após o término dos 10 atendimentos, as voluntárias foram reavaliadas quanto a dor lombar e as oscilações corporais conforme a avaliação inicial. Na Figura 1, demonstram-se alguns dos exercícios realizados. 
Figura 1. (A)-Fortalecimento da Musculatura do Assoalho Pélvico e Membros inferiores; (B) Propriocepção lombar associado à rotação de tronco; (C) Relaxamento lombar; (D)Alongamento de cadeia posterior.

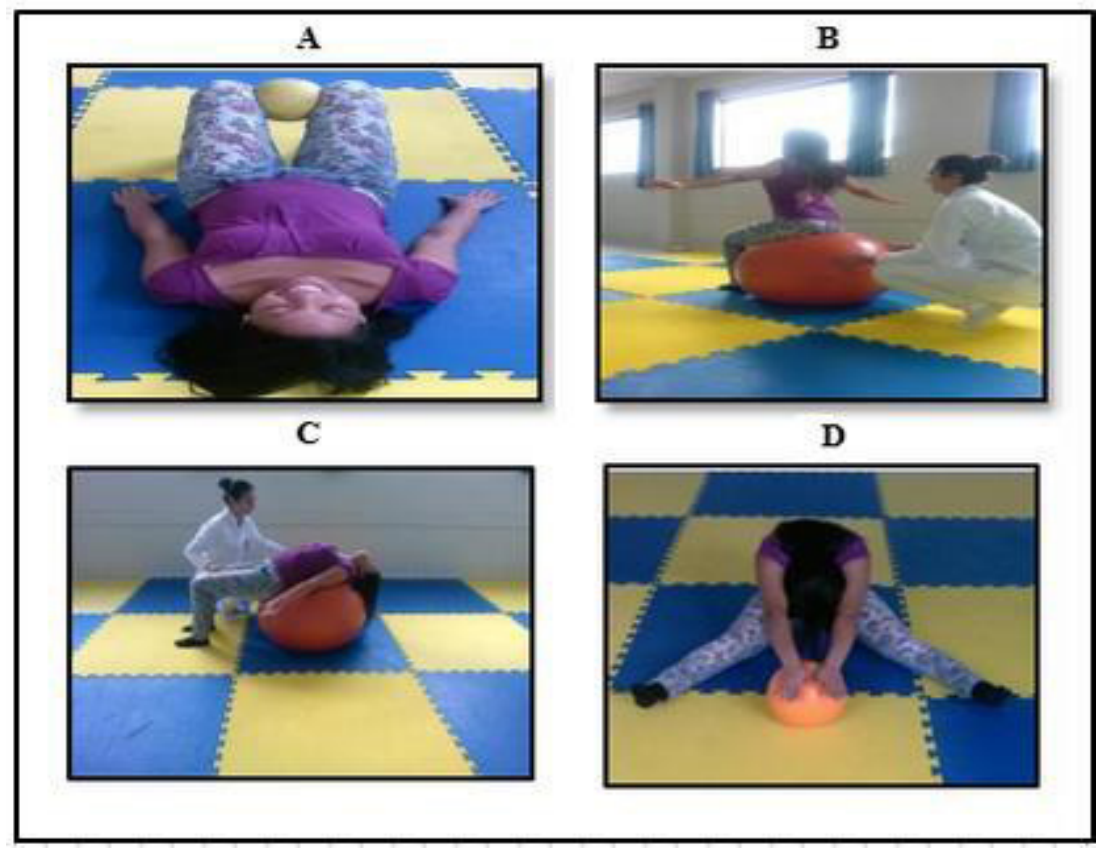

O Grupo Controle realizou os mesmos procedimentos de avaliação que o Grupo Pilates, porém não receberam nenhuma forma de atendimento e foi reavaliado após cinco semanas.

\section{RESULTADOS}

Utilizou-se o software Microsoft Excel 2010 para realização das tabelas e gráficos e o software BioEstat 5.3, para realização dos testes de normalidade e significância. Para testar a normalidade dos valores obtidos, utilizou-se o teste de Shapiro Wilk, com nível de significância de $95 \%$ ( $\mathrm{p} \leq 0,05)$. Como os dados obtiveram comportamento paramétrico, realizou-se o teste T-Student para análise intra-grupo.

Observa-se no gráfico 1, a Oscilação Estabilométrica do Grupo Pilates e do Grupo Controle, antes e depois do protocolo de tratamento utilizado, os dados apresentaram comportamento paramétrico e observa-se que não houve diferença estatisticamente significante entre os valores da oscilação em ambos os grupos.

Figura 1 - Grupo Pilates e valores da oscilação antes $(3,68 \pm 7,45)$ e depois $(3,60 \pm 8,58)$, ( $\mathrm{p}=1,00)$; Grupo Controle e valores antes $(3,40 \pm 1,35)$ e depois $(3,71 \pm 1,24),(\mathrm{p}=0,17)$.

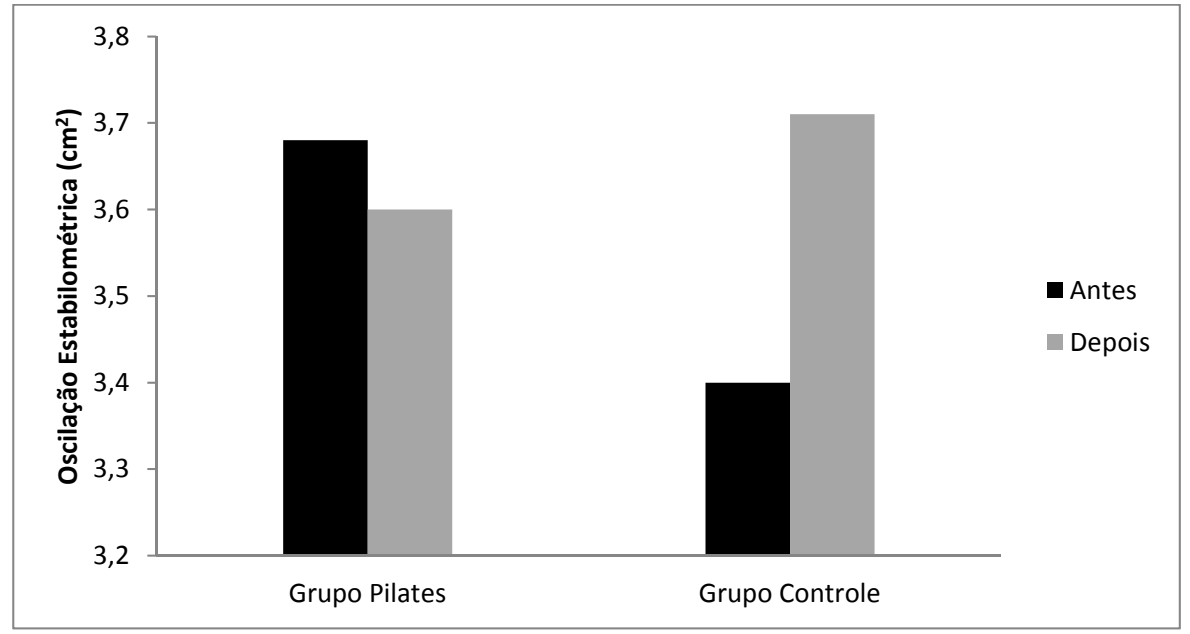


No gráfico 2, observam-se os valores da EVA do Grupo Pilates e do Grupo Controle, antes e depois do protocolo de tratamento utilizado, e observa-se que os dados obtiveram comportamento paramétrico e consequente realização do teste $t$ de Student, observou-se que houve diferença estatisticamente significante na redução dos valores da intensidade desse sintoma no grupo que realizou Pilates.

Figura 2 - Grupo Pilates e valores da EVA antes $(5,28 \pm 2,11)$ e depois $(1,42 \pm 1,04)$, ( $\mathrm{p}=0,0001)$; Grupo Controle e valores da EVA antes $(4,0 \pm 2,34)$ e depois $(4,6 \pm 2,39),(\mathrm{p}=0,1394) *$ Significância Estatística

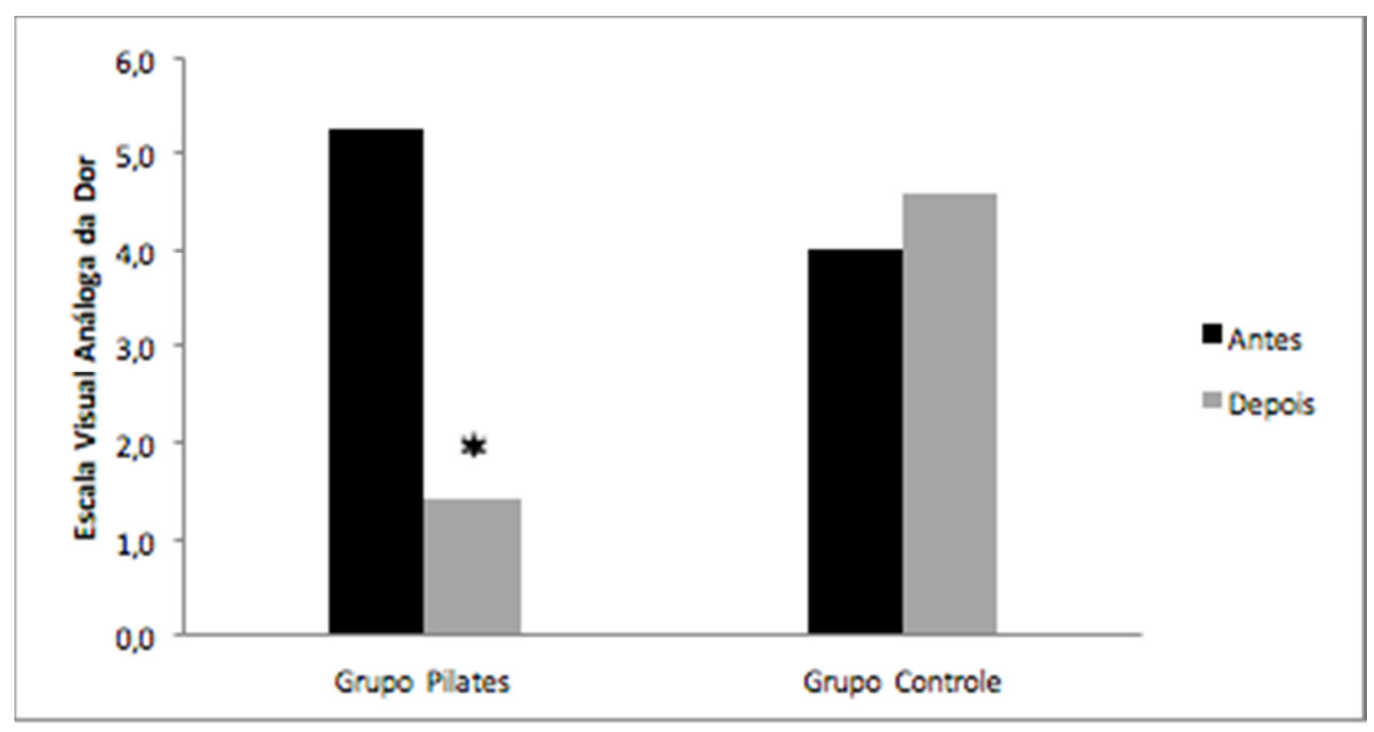

Para a comparação entre os grupos, foi realizado o cálculo do tamanho do efeito entre o Grupo Pilates e o grupo Controle, para isso utilizou-se o software SPSS, foi utilizado teste de ANOVA One Way, com teste de post-hoc de Tukey com nível de significância de 95\%. Essa análise demonstrou uma diferença estatisticamente significante na comparação da redução da dor pela EVA do grupo Pilates ( $p=0,001$ ) em relação ao grupo Controle. Evidenciando a eficácia do método Pilates na redução do quadro álgico das voluntárias. E em relação à variável Oscilação Estabilométrica não houve diferença estatisticamente significante $(\mathrm{p}=0,680)$, na comparação entre os grupos.

\section{DISCUSSÃO}

De acordo com os resultados do presente estudo, sugere-se que o método Pilates auxilia na redução da dor causada pelas alterações fisiológicas gestacionais, e que a oscilação do centro de massa de mulheres gestantes não é influenciada pelos exercícios do método. $\mathrm{O}$ presente estudo mostrou dificuldade em encontrar uma amostragem maior de mulheres gestantes para compor a análise, tendo em vista que muitas delas residiam longe da localidade onde a atividade foi realizada e/ou não tinham disponibilidade de tempo para participar.
Pode-se observar uma ampla prevalência de dor lombar durante o período gestacional, indo de encontro ao estudo de Firmento et. al., (2012), o qual descreveu que os principais motivos para a ocorrência da dor lombopélvica na gestação são as adaptações da coluna vertebral, que se deve, principalmente, à ação do hormônio relaxina e ao aumento considerável do peso do abdômen. Gomes et. at. (2013) concorda e acrescenta que, em decorrência do aumento da mobilidade nessas articulações, acentuam-se as exigências sobre os ligamentos e músculos estabilizadores, podendo ocasionar dor. Para Martins e Silva (2005), essas dores aumentam principalmente se a mulher apresentava esta queixa antes de engravidar e acrescentam que esse sintoma pode perdurar no período puerperal.

O presente estudo observou que as voluntárias que realizaram a atividade do Pilates obtiveram uma redução estatisticamente significante da dor em comparação com o Grupo Controle, enfatizando a importância do método Pilates na redução da intensidade de dor lombar, fato esse que se justifica pelo motivo de que o método proporciona um recrutamento de músculos profundos, fundamentais na estabilização pélvica e no controle biomecânico e funcional da coluna lombar, abordagem essa confirmada por Costa 
e Noronha, (2012), que em seu estudo de revisão da literatura, expõe que o método Pilates caracteriza-se por um conjunto de movimentos onde a posição neutra da coluna vertebral é sempre respeitada, objetivando a melhora da coordenação da respiração com o movimento do corpo, a flexibilidade geral, a força muscular e a postura, sendo, portanto, esses fatores essenciais no processo de reabilitação postural, e auxiliando na redução do quadro álgico, pela melhora e aprendizagem da estabilidade da coluna lombo-pélvica.

Já Vaz et. al., (2012), em seu estudo de revisão literária, citou que não existe correlação entre métodos que proporcionam ganho de flexibilidade muscular a diminuição da lombalgia, achados que justificam a redução da dor das gestantes avaliadas no presente estudo pela melhora da estabilidade dos músculos profundos, e não pelo ganho de flexibilidade. Mann et. al. (2008) discorda de Vaz et. al., (2012) quando, em seu estudo de revisão, descreve que há relação significativa entre a elasticidade da articulação sacroilíaca e a dor pélvica posterior.

Analisando os achados dos autores supracitados, acredita-se que métodos que proporcionem ganhos de flexibilidade em gestantes não devem ser aplicados, pois acentuariam a instabilidade mecânica proporcionando aumento da dor lombar gestacional (MARTINS, 2005).

Em relação ao Grupo Controle, observa-se nos resultados uma tendência ao aumento da dor das gestantes analisadas, mesmo que esse valor não tenha sido estatisticamente significante, pode ser justificada, pelo fato de que a inatividade física está diretamente relacionada a dores na coluna. Segundo Mann et. al. (2008), o sedentarismo, aliado à deficiência no sistema musculoesquelético e sobrecargas na coluna tornam os indivíduos propensos a dor lombar ou pélvica.

Quanto à oscilação do centro de massa das gestantes, constatou-se que não houve melhora significante na redução dessa oscilação para o Grupo Pilates $(p=1,00)$, enquanto que o Grupo Controle sugere um aumento da oscilação $(p=0,17)$, mesmo que esse aumento não tenha sido estatisticamente significante, sugere-se que essa tendência gráfica possa ser explicada pelo fato de que no Grupo Pilates as gestantes foram expostas a uma série de exercícios que visaram a mentalização e concentração na execução dos exercícios, focados na melhora da consciência corporal, proporcionando ganhos sintomáticos e não funcionais.
Hyun, Hwangbo e Lee (2014) corroboram com essa explicação quando, em seu estudo realizado com 40 idosas do sexo feminino, dizem que a atividade dos músculos do tronco mantém o equilíbrio contra a gravidade, ajusta postura, e prepara-se para o movimento das extremidades nas atividades de vida diária, gerando ganhos muitas vezes profundos em músculos estabilizadores, porém sem ganhos biomecânicos funcionais.

Bankoff et. al., (2004) descreveu a importância de alguns fatores para melhorar e manter o equilíbrio corporal como, por exemplo, praticar atividades físicas diariamente, realizar exercícios respiratórios, de alongamento e relaxamento. Ashrafinia et. al., (2014) concorda e ainda descreve o método Pilates como grande beneficiador para tais finalidades, devido ao fato de proporcionar às gestantes conhecerem melhor o seu corpo e se sentirem preparadas e confiantes em si mesmas. A praticante reorganiza o seu centro de força através de uma prática variada com poucas repetições.

Mann et. al. (2011) e Silva e Tufanin (2013) observaram que, com o avançar da gestação, as oscilações corporais têm um agravante, as alterações advindas de mudanças biomecânicas e hormonais, como o constante crescimento do útero, o aumento no peso corporal e o tamanho das mamas, que contribuem para o deslocamento do centro de gravidade, acentuando a anteversão pélvica e promovendo um consequente desequilíbrio. Além, de relatar que há uma compensação da hiperlordose lombar, de forma que as gestantes passam a utilizar base de suporte maior e modificações nos padrões considerados normais para a marcha e para o equilíbrio, porém, o presente estudo não comparou períodos gestacionais diferentes para poder discutir de forma mais aprofundada com o autor acima citado.

Acredita-se que o baixo número de gestantes incluídas no trabalho, bem como a dificuldade de encontrar gestantes dispostas e possibilitadas a realizar exercícios tenha sido as principais limitações do presente estudo, bem como as dores e desconfortos encontrados durante o período gestacional possam ser pontuados como possíveis fatores limitantes na execução do trabalho. Contudo, sugere-se que novos trabalhos sejam realizados, com um número maior de gestantes, e correlacionando mais variáveis além da oscilação do centro de massa do corpo. 


\section{CONCLUSÕES}

O presente estudo demonstrou que um programa de Pilates solo, aplicado em gestantes entre o $3^{\circ}$ e $8^{\circ}$ mês de gestação, realizando exercícios duas vezes por semana, durante cinco semanas, reduziu a dor lombar em relação ao grupo controle. Entretanto, os achados demonstraram que 10 atendimentos não foram suficientes para gerar alterações do centro de oscilação corporal.

\section{REFERÊNCIAS}

ALBINO, M. A. S.; MOCCELLIN, A. S.; FIRMENTO, B. S.; DRIUSSO, P. Modificações da força de propulsão da marcha durante a gravidez: efeito das alterações nas dimensões dos pés. Rev. Bras. Ginecol. Obstet. vol.33 no.7 Rio de Janeiro July 2011.

ASHRAFINIA, F.; MIRMOHAMMADALI, M.; RAJABI, H.; KAZEMNEJAD, A. SADEGHNIIATHAGHIGHI, K.; AMELVALIZADEH, M.; CHEN, HUI. The effects of Pilates exercise on sleep quality in postpartum women. Journal of Bodywork \& Movement Therapies, 18, 190$199 ; 2014$

BANKOFF, A. D. P.; CIOL, P.; ZAMAI, C. A.; SCHMIDT, A.; BARROS, D. D. ESTUDO DO EQUILÍBRIO CORPORAL POSTURAL ATRAVÉS DO SISTEMA DE BAROPODOMETRIA ELETRÔNICA. Revista Conexões, v.2, n.2, 2004.

BARBOSA, C. M. S.; SILVA, J. M. N.; MOURA, A. B. Correlação entre o ganho de peso e a intensidade da dor lombar em gestantes. Rev. Dor. vol.12 no.3 São Paulo Jul/ Set. 2011

CORTEZ, P. J. O.; FRANCO, T. A. S.; SENE, T. M.; CARVALHO, T. D.; TOMAZINI, J. E. Correlação entre a dor lombar e as alterações posturais em gestantes. Arquivos Brasileiros de Ciências da Saúde, v.37, n. 1, p. 30-35, Jan/ Abr 2012.

COSTA, L. M. R.; NORONHA, M. O método pilates no Brasil: uma revisão de literatura. Arq. Catarin. Med.; 41 (3): 87-92; 2012.

DUARTE, M. ANÁLISE ESTABILOGRAFICA DA POSTURA ERETA HUMANA QUASE-ESTÁTICA. Tese: Escola de Educação Fisica e Esporte - Universidade de São Paulo. 2000.

FERREIRA, A. S.; GAVE, N. S.; ABRAHÃO, F.; SILVA, J. G. Influencia da morfologia dos pés no equilíbrio durante o apoio bipodal. Fisioter. Mov., Curitiba, v.23, n. 2, p. 193200, abr./jun. 2010.

FIRMENTO, B. S.; MOCCELLIN A. S.; ALBINO, M. A. S.; DRIUSSO, P. Avaliação da lordose lombar e sua relação com a dor lombopélvica em gestantes. Fisioter Pesq. 19(2):128-34; 2012.

GOMES, M. R. A.; ARAUJO, R. C.; LIMA, A. S.; PITANGUI, A. C. R. Lombalgia gestacional: prevalência e características clínicas em um grupo de gestantes. Rev Dor. São Paulo, 14 (2): 114-7; abr-jun 2013.

HYUN, JU.; HWANGBO, KAK.; LEE, CHAE-WOO. The Effects of Pilates Mat Exercise on the Balance Ability of Elderly Females. J. Phys. Ther. Sci., Vol. 26, No. 2, 2014.

LIMA, A. S.; GOMES, M. R. A.; ARAÚJO, R. C.; PITANGUI, A. C. R. Análise da postura e frequência de lombalgia em gestantes: estudo piloto. J Health Sci Inst. V. 29 ed. 4 ; 290-300. 2011.

MANN, L.; KLEINPAUL, J. F.; TEIXEIRA, C. S.; MOTA, C. B. Influencia dos Sistemas Sensoriais na Manutenção do Equilíbrio em Gestantes. Fisioter. Mov., Curitiba, v. 24, n. 2, p. 315-325, abr./jun. 2011.

MANN, L.; KLEINPAUL, J. J.; TEIXEIRA, C. S.; KONOPKA, C. K. DOR LOMBO-PÉLVICAEEXERCÍCIO FÍSICO DURANTE A GESTAÇÃO. Fisioter. Mov. 21(2): 99-105; abr/jun; 2008.

MARÉS, G.; OLIVEIRA, K. B.; PIAZZA, M. C.; PREIS, C.; NETO, L. B. A importância da estabilização central no método Pilates: uma revisão sistemática. Fisioter. Mov., Curitiba, v. 25, n. 2, p. 445-451, abr./jun. 2012.

MARTINS, R. F.; SILVA, J. L. P. Tratamento da lombalgia e dor pélvica posterior na gestação por um método de exercícios. Rev Bras Ginecol Obstet. 27 (5): 275-82; 2005.

NASCIMENTO, S. L.; GODOY, A. C.; SURITA, F. G.; PINTO e SILVA L. Recomendações para prática de exercício físico na gravidez: uma revisão critica da literatura. Rev Bras Ginecol Obstet., Rio de Janeiro, v. 36, n. 9, set. 2014.

NOVAES, F. S.; SHIMO, A.K. K.; LOPES, M. H. B. M. Lombalgia na gestação. Rev. Latino-Am. Enfermagem vol.14 no.4 Ribeirão Preto July/Aug. 2006.

POSADZKI, P.; LIZIS, P.; DERENGOWSKA, M. H. Pilates for low back pain: A systematic review. Complementary Therapies in Clinical Practice, 17, 85-89, 2011.

RIBAS SI E GUIRRO ECO. Análise da pressão plantar e do equilibrio postural em diferentes fases da gestação. Rev. bras. fisioter. São Carlos, v. 11, n. 5, p. 391-396, set./out. 2007.

RODRIGUES, B. G. S.; CADER, S. A.; OLIVEIRA, E. M.; TORRES, N. V. O. B.; DANTAS. E. H. M. Avaliação do equilíbrio estático de idosas pós-treinamento com método pilates. R. bras. Ci. e Mov., 17(4):25-33, 2009.

RODRIGUES, B. G. S.; CADER, S. A.; TORRES, N. V. O. B.; OLIVEIRA, E. M.; DANTAS, E. H. M. Autonomia funcional de idosas praticantes de Pilates. Fisioterapia e Pesquisa, São Paulo, v.17, n.4, p.300-5, out/dez.2010. 
SILVA, A. C. L. G.; MANNRICH, G. PILATES NA REABILITAÇÃO: uma revisão sistemática. Fisioter. Mov., Curitiba, v. 22, n. 3, p. 449-455, jul./set. 2009.

SILVA, C. S.; TUFANIN, A. T. Alterações respiratórias e biomecânicas durante o terceiro trimestre de gestação: uma revisão de literatura. Revista Eletrônica Saúde e Ciência. Volume III; Nro 2; ISSN 2238-4111, 2013.

VAZ, R. A.; LIBERALI, R.; CRUZ, T. M. F.; NETTO, M. I. A. O método pilates na melhora da flexibilidade - Revisão Sistemática. Revista Brasileira de Prescrição e Fisiologia do Exercício, São Paulo, v.6, n.31, p.25-31. Jan/Fev. 2012. 